\title{
Polyarteritis nodosa
}

\section{presenting with catastrophic involvement of the intra- abdominal arterial tree}

Received: 31 October, 2020

Accepted: 23 January, 2021

Published: 27 January, 2021

*Corresponding author: Gwenno Edwards, MBBCh, MRCP, Department of Nephrology, Ysbyty Gwynedd, Bangor, Wales, E-mail: gwenno.edwards3@wales. nhs.uk; gwenno.m@hotmail.co.uk

Keywords: Polyarteritis nodosa

https://www.peertechz.com

Check for updates

\author{
Gwenno Edwards ${ }^{1 *}$, Abdulfattah Alejmi ${ }^{1}$ and Janice Harper ${ }^{2}$ \\ ${ }^{1} \mathrm{MBBCh}, \mathrm{MRCP}$, Department of Nephrology, Ysbyty Gwynedd, Bangor, Wales \\ ${ }^{2} \mathrm{MBBCh}, \mathrm{MRCP}$, Department of Nephrology, Royal Liverpool University Hospital, Liverpool, England
}

\begin{abstract}
We report a case of a 63-year-old female presenting with abdominal pain, who suffered a dramatic deterioration in her condition following a diagnosis of bilateral renal infarcts on imaging. Within days she had developed widespread aneurysmal dilatation, dissection and thrombosis of the intra-abdominal arterial tree, requiring emergency transfer to a tertiary vascular centre for treatment of polyarteritis nodosa. She responded well to intravenous cyclophosphamide and is now receiving treatment on an outpatient basis.
\end{abstract}

\section{Background}

Flank pain secondary to renal infarction is an uncommon presentation of polyarteritis nodosa. As a result, the diagnosis can be missed in cases where this is the sole manifestation. Patients can deteriorate rapidly and present late with gastro-intestinal bleeding and thrombosis therefore a high index of suspicion is required. Prompt treatment with immunosuppressive therapy has dramatically decreased mortality [1].

\section{Case report}

A 63-year-old female patient presented with a oneweek history of abdominal pain associated with intermittent vomiting and generalised myalgias. She was taking Edoxaban for atrial fibrillation. On physical examination her blood pressure was $146 / 86 \mathrm{mmHg}$ and she had right-sided loin and lower quadrant tenderness.

Her blood tests demonstrated normal haemoglobin, normal renal function and a C-reactive protein of $60 \mathrm{mg} / \mathrm{L}$. Hepatitis $\mathrm{B}$ surface antigen was negative. Abdominal and chest $\mathrm{X}$-rays were normal. The admitting surgeons diagnosed diverticulitis and organised a Computed Tomography (CT)-scan of her abdomen and pelvis. The CT-scan demonstrated bilateral renal infarcts and a pancreatic infarct of unclear cause. It was thought initially that her infarcts were as a result of thromboembolism secondary to atrial fibrillation. The patient was transferred to the medical ward and commenced on an intravenous heparin infusion. Edoxaban was discontinued.

Three days into her admission the patient developed worsening abdominal pain. Physical examination revealed abdominal guarding and a blood pressure of 220/100mmHg. A repeat CT-scan demonstrated a dramatic deterioration in the appearances with extensive vascular changes. Widespread aneurysms to the branches of the intra-abdominal arterial tree were seen with multiple occlusions to the distribution of the coeliac, superior and inferior mesenteric artery networks. The appearances were interpreted as an evolving catastrophic vascular pathology (Figure 1). Her repeat laboratory tests showed a drop in haemoglobin from $138 \mathrm{~g} / \mathrm{L}$ to $86 \mathrm{~g} / \mathrm{L}$, a creatinine of $141 \mathrm{micromol} / \mathrm{L}$ and a C-reactive protein of $278 \mathrm{mg} / \mathrm{L}$.

The patient deteriorated significantly the following day, developing refractory hypotension at $70 / 50 \mathrm{mmHg}$ despite intravenous fluid resuscitation. A further CT-scan demonstrated intra-abdominal haemorrhage $(21 \times 7 \times 7 \mathrm{~cm})$. 


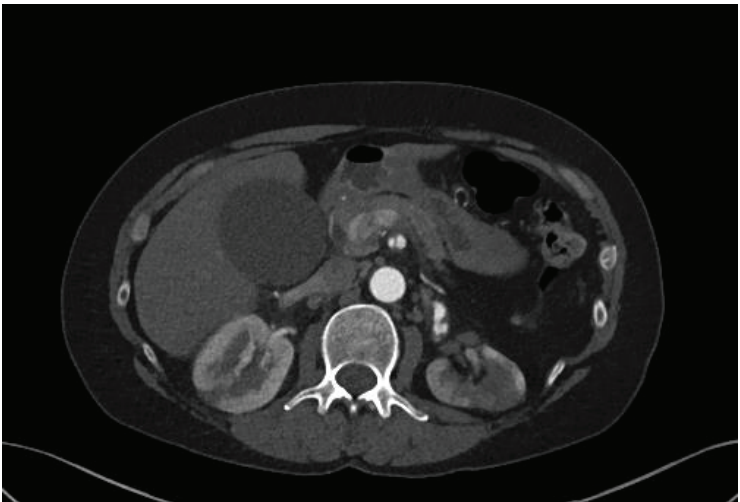

Figure 1: Dissection of the superior mesenteric artery with renal artery aneurysm and adjacent dissection.

The patient was immediately commenced on intravenous methylprednisolone on the basis of a clinical diagnosis of polyarteritis nodosa. She was transferred to the local tertiary unit for specialist vasculitis care and was commenced on intravenous cyclophosphamide. She received blood transfusions and her systolic blood pressure was maintained at $<120 \mathrm{mmHg}$.

The patient made a remarkable recovery and was discharged with 6 months of outpatient intravenous cyclophosphamide induction treatment. She was commenced on oral prednisolone and a therapeutic dose of low-molecular-weight-heparin. After 6 months the patient was converted to azathioprine maintenance treatment and a reducing dose of prednisolone regimen. She was also converted from low-molecular-weightheparin to Apixaban.

The patient is now on azathioprine monotherapy and remains asymptomatic 10 months on.

\section{Discussion}

Polyarteritis Nodosa (PAN) is defined as a necrotizing arteritis of medium or small arteries without glomerulonephritis or vasculitis in arterioles, capillaries, or venules, and not associated with Antineutrophil Cytoplasmic Antibodies (ANCAs). ANCA is a useful discriminator, as PAN and ANCAassociated vasculitis can be clinically and pathologically indistinguishable. A diagnosis of PAN can be made if a patient exhibits three of the ten classifiers outlined by the American College of Rheumatology [1].

PAN is rare, affecting approximately 1.5 per 1 million population. It affects males and females equally and is found in all races. The age at onset is usually between 40 and 60 years. The aetiology is unknown and likely to be diverse. It is proposed that immune complexes are triggered which initiate inflammation but the mechanism is unclear. A minority of patients have hepatitis B virus infection [1].

Contrast angiography is considered the gold-standard for identifying the vascular abnormalities found in PAN; however this carries significant risks such as bleeding and embolization. In situations where angiography is deemed too dangerous, CT or MR angiography is advised [2,3].

Untreated PAN is associated with a high mortality rate, with GI bleeding secondary to bowel ischaemia being one of the leading causes of death [1]. In non-hepatitis B virus-related PAN the mainstay of treatment is long-term immunosuppressive therapy, however these therapies carry the risk of significant side effects [1]. In those with severe disease, survival rates are better in patients treated with both high-dose cyclophosphamide and glucocorticoids [4]. Patients who have completed their induction treatment should be switched to a maintenance immunosuppressive agent such as azathioprine or methotrexate. The above recommendations are based on trials in ANCA-associated vasculitides [5].

\section{Conclusion}

This case highlights an unusual presentation of acute renal infarction in an anti-coagulated patient in whom we witnessed a dramatic and sudden deterioration. It is very likely that, without prompt immunosuppressive treatment, her condition would have been fatal [1]. As a result of the quick actions of her medical team the patient made a full recovery with normalisation of her renal function.

We suggest that a diagnosis of polyarteritis nodosa should be considered in any patient presenting with bilateral renal infarction of unknown cause.

\section{Acknowledgments}

We are indebted to consultant radiologist Dr Hugh Godfrey who provided us with the image.

\section{References}

1. Pagnoux C, Seror R, Henegar C, Mahr A, Cohen P, et al. (2010) French Vasculitis Study Group. Clinical features and outcomes in 348 patients with polyarteritis nodosa: a systematic retrospective study of patients diagnosed between 1963 and 2005 and entered into the French Vasculitis Study Group Database. Arthritis Rheum 62: 616-626. Link: https://bit.ly/3psxLKI

2. Farrah, TE, Basu E, Dweck M, Calcagno C, Fayad ZA, et al. (2019) Advances in Therapies and Imaging for Systemic Vasculitis. Arterioscler Thromb Vasc Bio 39: 1520-1541. Link: https://bit.ly/2Mjuyif

3. Dhaun N, Patel D, Kluth DC (2013) Computed tomography angiography in the diagnosis of ANCA-associated small and medium vessel vasculitis. Am $J$ Kidney Dis 62: 390-393. Link: https://bit.ly/2Yc66SE

4. Gayraud M, Guillevin L, le Toumelin P, Cohen P, Lhote F, et al. (2001) Long-term follow up of polyarteritis nodosa, microscopic polyangiitis, and Churg-Strauss syndrome: analysis of four prospective trials including 278 patients. Arthritis Rheum 44: 666. Link: https://bit.ly/39h8XQx

5. Jayne D, Rasmussen N, Andrassy K, Bacon P, Tervaert JW, et al. (2003) A randomized trial of maintenance therapy for vasculitis associated with antineutrophil cytoplasmic antibodies. N Engl J Med 349: 36-44. Link: https://bit.ly/2Y9ACMC

Copyright: @ 2021 Edwards G, et al. This is an open-access article distributed under the terms of the Creative Commons Attribution License, which permits unrestricted use, distribution, and reproduction in any medium, provided the original author and source are credited. 